[Article]

\title{
以类水滑石为前驱体的 $\mathrm{Cu} / \mathrm{Zn} / \mathrm{Al} /(\mathrm{Zr}) /(\mathrm{Y})$ 催化剂制备及其催化 $\mathrm{CO}_{2}$ 加氢合成甲醇的性能
}

\author{
高 鹏 ${ }^{1,2}$ 李 枫 $^{2}$ 赵 宁 ${ }^{2, *}$ 王 慧 ${ }^{1}$ 魏 伟 ${ }^{1, *}$ 孙予罕 ${ }^{2,3}$
}

('中国科学院上海高等研究院, 温室气体与环境工程研究中心, 上海 201203; '2中国科学院山西煤炭化学研究所, 煤转化国家 重点实验室, 太原 $030001 ; 3$ 中国科学院上海高等研究院, 中国科学院低碳转化科学与工程重点实验室, 上海 201203)

\begin{abstract}
摘要: 采用共沉淀法合成了 Cu:Zn:Al:Zr:Y原子比分别为 2:1:1:0:0、2:1:0.8:0.2:0、2:1:0.8:0:0.2 和 2:1:0.8:0.1: 0.1 的 $\mathrm{Cu} / \mathrm{Zn} / \mathrm{Al} /(\mathrm{Zr}) /(\mathrm{Y})$ 类水滑石化合物. 将前驱体材料在空气中 $500{ }^{\circ} \mathrm{C}$ 焙烧后得到复合金属氧化物, 并将其 用于 $\mathrm{CO}_{2}$ 加氢合成甲醇反应. 采用 $X$ 射线衍射 $(X R D)$ 、热重 $(T G)$ 分析、 $N_{2}$ 吸附、氧化亚氮 $\left(\mathrm{N}_{2} \mathrm{O}\right)$ 反应吸附、氢气程 序升温还原 $\left(\mathrm{H}_{2}-\mathrm{TPR}\right)$ 和 $\mathrm{H}_{2} / \mathrm{CO}_{2}$ 程序升温脱附 $\left(\mathrm{H}_{2} / \mathrm{CO}_{2}-\mathrm{TPD}\right)$ 技术对所制备的样品进行表征. 结果表明, $\mathrm{Zr}$ 和 $\mathrm{Y}$ 的引入使得催化剂BET 比表面积大幅增加, 金属铜的比表面积和分散度均按以下顺序依次增加: Cu/Zn/AK< $\mathrm{Cu} / \mathrm{Zn} / \mathrm{Al} / \mathrm{Zr}<\mathrm{Cu} / \mathrm{Zn} / \mathrm{Al} / \mathrm{Y}<\mathrm{Cu} / \mathrm{Zn} / \mathrm{Al} / \mathrm{Zr} / \mathrm{Y}$, 然而, 强碱位数目占总碱位数目的比例的变化顺序为: $\mathrm{Cu} / \mathrm{Zn} / \mathrm{Al}<\mathrm{Cu} /$ $\mathrm{Zn} / \mathrm{Al} / \mathrm{Y}<\mathrm{Cu} / \mathrm{Zn} / \mathrm{A} / / \mathrm{Zr} / \mathrm{Y}<\mathrm{Cu} / \mathrm{Zn} / \mathrm{Al} / \mathrm{Zr}$. 活性评价结果揭示 $\mathrm{CO}_{2}$ 转化率取决于金属铜的比表面积, 甲醇选择性则 随强碱位比例的增加而线性增加. 因而, $Z r$ 和 $Y$ 的引入有利于甲醇的合成, $C u / Z n / A / / Z r / Y$ 催化剂上的甲醇收率 最高.
\end{abstract}

关键词： 类水滑石前驱体；助剂；Cu/Zn/Al催化剂；二氧化碳加氢； 甲醇 中图分类号: 0643

\section{Preparation of $\mathrm{Cu} / \mathrm{Zn} / \mathrm{Al} /(\mathrm{Zr}) /(\mathrm{Y})$ Catalysts from Hydrotalcite-Like Precursors and Their Catalytic Performance for the Hydrogenation of $\mathrm{CO}_{2}$ to Methanol}

\author{
GAO Peng $\quad$ LI Feng ${ }^{1,2}$ ZHAO Ning, $\quad$ WANG Hui $\quad$ WEI Wei ${ }^{1, *} \quad$ SUN Yu-Han ${ }^{2,3}$ \\ ('Center for Greenhouse Gas and Environmental Engineering, Shanghai Advanced Research Institute, Chinese Academy of Sciences, \\ Shanghai 201203, P. R. China; ${ }^{2}$ State Key Laboratory of Coal Conversion, Institute of Coal Chemistry, Chinese Academy of \\ Sciences, Taiyuan 030001, P. R. China; ${ }^{3}$ Chinese Academy of Sciences Key Laboratory of Low-Carbon Conversion Science \\ and Engineering, Shanghai Advanced Research Institute, Chinese Academy of Sciences, Shanghai 201203, P. R. China)
}

\begin{abstract}
Cu} / \mathrm{Zn} / \mathrm{Al} /(\mathrm{Zr}) /(\mathrm{Y})$ hydrotalcite-like compounds with $\mathrm{Cu}: \mathrm{Zn}: \mathrm{Al}: \mathrm{Zr}: Y$ atomic ratios of 2:1:1:0:0, 2:1: 0.8:0.2:0, 2:1:0.8:0:0.2, and 2:1:0.8:0.1:0.1 were prepared using the coprecipitation method. The mixed oxides were then obtained by the calcination of the precursors at $500{ }^{\circ} \mathrm{C}$ in air, and subsequently evaluated in terms of their catalytic performance for the synthesis of methanol from the hydrogenation of $\mathrm{CO}_{2}$. The asprepared samples were characterized by X-ray diffraction (XRD), thermogravimetric (TG) analysis, $\mathrm{N}_{2}$ adsorption, reactive $\mathrm{N}_{2} \mathrm{O}$ adsorption, $\mathrm{H}_{2}$ temperature-programmed reduction $\left(\mathrm{H}_{2}-\mathrm{TPR}\right)$, and $\mathrm{H}_{2} / \mathrm{CO}_{2}$ temperature-programmed desorption $\left(\mathrm{H}_{2} / \mathrm{CO}_{2} \mathrm{TPD}\right)$ techniques. The results of these analyses showed that the BET specific surface area increased significantly with the introduction of $\mathrm{Zr}$ and $\mathrm{Y}$, which was related to
\end{abstract}

Received: February 10, 2014; Revised: April 1, 2014; Published on Web: April 2, 2014.

"Corresponding authors. ZHAO Ning, Email: zhaoning@sxicc.ac.cn; Tel: +86-351-4049612. WEI Wei, Email: weiwei@sari.ac.cn;

Tel: +86-21-20608001.

The project was supported by the National Key Technology Research and Development Program of the Ministry of Science and Technology, China (2013BAC11B02) and Strategic Priority Research Program of the Chinese Academy of Sciences (XDA05010109, XDA05010110, XDA05010204). 科技部国家科技支撑计划(2013BAC11B02)和中国科学院战略性先导科技专项(XDA05010109, XDA05010110, XDA05010204)资助项目

(C) Editorial office of Acta Physico-Chimica Sinica 
the amount of $\mathrm{H}_{2} \mathrm{O}$ and $\mathrm{CO}_{2}$ evolved from the precursors during calcination. The $\mathrm{Cu}$ specific surface area and $\mathrm{Cu}$ dispersion properties increased in the order of $\mathrm{Cu} / \mathrm{Zn} / \mathrm{Al}<\mathrm{Cu} / \mathrm{Zn} / \mathrm{Al} / \mathrm{Zr}<\mathrm{Cu} / \mathrm{Zn} / \mathrm{Al} / \mathrm{Y}<\mathrm{Cu} / \mathrm{Zn} / \mathrm{Al} / \mathrm{Zr} / \mathrm{Y}$, whereas the proportion of strongly basic sites to total basic sites increased in the order of $\mathrm{Cu} / \mathrm{Zn} / \mathrm{Al}<\mathrm{Cu} / \mathrm{Zn} /$ $\mathrm{Al} / \mathrm{Y}<\mathrm{Cu} / \mathrm{Zn} / \mathrm{Al} / \mathrm{Zr} / \mathrm{Y}<\mathrm{Cu} / \mathrm{Zn} / \mathrm{Al} / \mathrm{Zr}$. The evaluation of these materials as catalysts for the hydrogenation of $\mathrm{CO}_{2}$ revealed that the $\mathrm{CO}_{2}$ conversion was dependent on the $\mathrm{Cu}$ specific surface area, and the $\mathrm{CH}_{3} \mathrm{OH}$ selectivity increased linearly as the proportion of strongly basic sites increased. The introduction of $\mathrm{Zr}$ and $\mathrm{Y}$ therefore favored the production of methanol and the maximum $\mathrm{CH}_{3} \mathrm{OH}$ yield was obtained over the $\mathrm{Cu}$ / $\mathrm{Zn} / \mathrm{Al} / \mathrm{Zr} / \mathrm{Y}$ catalyst.

Key Words: Hydrotalcite-like precursor; Modifier; $\mathrm{Cu} / \mathrm{Zn} / \mathrm{Al}$ catalyst; $\mathrm{CO}_{2}$ hydrogenation; Methanol

\section{1 引 言}

大气中 $\mathrm{CO}_{2}$ 浓度增加导致的温室效应与化石然 料的圆乏正日趋受到世界范围的关注. 将 $\mathrm{CO}_{2}$ 转化 为有价值的化学品或燃料是解决温室气体控制以 及缓解能源圆乏的重要途径. ${ }^{1} \mathrm{CO}_{2}$ 可以转化为许多 物质, 在诸多的可能产物中, 甲醇成为很多研究者 首选的目标产物, 这是因为甲醇既是重要的化工原 料, 又是非石油基洁净合成然料. 甲醇还可以转化 为烯烃、汽油、柴油和芳烃, 从石油或天然气得到的 产品几乎都可以通过甲醇生产. ${ }^{2}$ 另外, 相对于传统 的合成气生产甲醇工艺, $\mathrm{CO}_{2}$ 加氢合成甲醇过程中 副产物更少, 使得后续精馏过程更简单, 有利于甲 醇的进一步化学转化. ${ }^{3}$

工业上, 以含有 $\mathrm{CO}_{2}$ 的合成气为原料, 采用 $\mathrm{Cu} /$ $\mathrm{Zn} / \mathrm{Al}$ 催化剂进行甲醇合成, 所以, $\mathrm{Cu} / \mathrm{Zn} / \mathrm{Al}$ 催化剂 在 $\mathrm{CO}_{2}$ 加氢合成甲醇反应中也受到了广泛研究. ${ }^{4-8}$ 通过添加某些金属助剂, 可以进一步提高 $\mathrm{Cu} / \mathrm{Zn} / \mathrm{Al}$ 催化剂的反应性能. $\mathrm{Zr}$ 的引入可以提高 $\mathrm{Cu}$ 的分散 度以及表面碱性, 从而改善催化剂对 $\mathrm{H}_{2}$ 和 $\mathrm{CO}_{2}$ 的吸 附性能. ${ }^{9,10}$ 另外, 有报道称 $\mathrm{Y}$ 的加入可以大幅提高催 化剂的比表面积, ${ }^{11}$ 但是有关 $\mathrm{Y}$ 助剂对于 $\mathrm{CO}_{2}$ 加氢合 成甲醇反应性能影响的报道较少. 除了催化剂组 分, 前驱体的结构对催化剂的物理化学性质也起着 非常重要的作用. 复合金属氧化物可以通过控制类 水滑石 $(\mathrm{HTl})$ 化合物热分解获得, 类水滑石化合物的 化学组成具有如下通式: $\left[\mathrm{M}_{1-x}^{2+} \mathrm{M}_{x}^{3+}(\mathrm{OH})_{2}\right]^{x+}\left(\mathrm{A}^{n-}\right)_{x / n}$. $m \mathrm{H}_{2} \mathrm{O}$, 其中 $\mathrm{M}^{2+}$ 和 $\mathrm{M}^{3+}$ 分别为二价和三价金属阳离 子, 位于主体层板上; $\mathrm{A}^{n-}$ 为层间阴离子. ${ }^{12}$ 以类水滑 石材料为焙烧前驱体制备的复合金属氧化物具有 阴阳离子易调变、比表面积高、金属元素分布均匀、 热稳定性高、以及较强的碱性等优点. ${ }^{13,14}$ 因而, 以类 水滑石为催化剂的前驱体有利于 $\mathrm{Cu} / \mathrm{Zn} / \mathrm{Al}$ 催化剂 反应性能的提高.

本文合成了一系列 Zr、Y 以及 $\mathrm{Zr}$ 和 $\mathrm{Y}$ 共同修饰
的 $\mathrm{Cu} / \mathrm{Zn} / \mathrm{Al}$ 类水滑石化合物, 焙烧后得到 $\mathrm{Cu} / \mathrm{Zn} / \mathrm{Al} /$ $(\mathrm{Zr}) /(\mathrm{Y})$ 催化剂并用于 $\mathrm{CO}_{2}$ 加氢合成甲醇的反应. 对 比考察了 Zr、Y 助剂对类水滑石前驱体材料的结构 和催化剂物理化学性质的影响, 以及两种助剂的协 同作用. 此外, 还对前驱体结构、催化剂物理化学性 质以及催化性能三者之间的关系进行了研究.

\section{2 实验部分}

\section{1 催化剂的制备}

实验所用试剂为分析纯的 $\mathrm{Cu}\left(\mathrm{NO}_{3}\right)_{2} \cdot 3 \mathrm{H}_{2} \mathrm{O}$ 、 $\mathrm{Zn}\left(\mathrm{NO}_{3}\right)_{2} \cdot 6 \mathrm{H}_{2} \mathrm{O} 、 \mathrm{Al}\left(\mathrm{NO}_{3}\right)_{3} \cdot 9 \mathrm{H}_{2} \mathrm{O}, \mathrm{ZrO}\left(\mathrm{NO}_{3}\right)_{4} \cdot 2 \mathrm{H}_{2} \mathrm{O}$ 、 $\mathrm{Y}\left(\mathrm{NO}_{3}\right)_{3} \cdot 6 \mathrm{H}_{2} \mathrm{O}$ 、无水 $\mathrm{NaOH}$ 和 $\mathrm{Na}_{2} \mathrm{CO}_{3}$. 四种催化剂 组成 $(\mathrm{Cu}: \mathrm{Zn}: \mathrm{Al}: \mathrm{Zr}: Y$ 原子比) 分别为 $2: 1: 1: 0: 0,2: 1$ : 0.8:0.2:0, 2:1:0.8:0:0.2 和 2:1:0.8:0.1:0.1. 将各种金属 的硝酸盐及硝酸氧盐按上述比例配制成一定浓度 的混合盐溶液, 以氢氧化钠和碳酸钠的混合水溶液 为碱溶液. 在室温摚拌条件下, 将混合盐溶液和碱 溶液加入到沉淀槽中, $\mathrm{pH}$ 值保持 10 . 沉淀结束后在 搅拌状态下于 $60^{\circ} \mathrm{C}$ 老化 $15 \mathrm{~h}$, 经洗涤、过滤后, 于 $100{ }^{\circ} \mathrm{C}$ 干燥 $12 \mathrm{~h}, 500{ }^{\circ} \mathrm{C}$ 焙烧 $4 \mathrm{~h}$, 降至室温后, 压 片、破碎至 40-60目. 得到的 $\mathrm{Cu} / \mathrm{Zn} / \mathrm{Al} 、 \mathrm{Cu} / \mathrm{Zn} / \mathrm{Al} / \mathrm{Zr}$ 、 $\mathrm{Cu} / \mathrm{Zn} / \mathrm{Al} / \mathrm{Y}$ 和 $\mathrm{Cu} / \mathrm{Zn} / \mathrm{Al} / \mathrm{Zr} / \mathrm{Y}$ 催化剂分别记作 CHTA、CHT-AZ、CHT-AY 和 CHT-AZY, 高温焙烧前, 相 应的焙烧前驱体材料分别记作 HT-A、HT-AZ、HTAY 和 HT-AZY.

\section{2 催化剂的表征}

催化剂的晶相结构测定在荷兰帕纳科公司生 产的 X'Pert Pro 型多晶X射线衍射仪上进行. 采用石 墨单色器, $\mathrm{Cu} K_{\mathrm{a}}$ 射线, $2 \theta$ 扫描范围为 $5^{\circ}-75^{\circ}$, 扫描步 长为 $0.0167^{\circ}$, 每步时间为 $12 \mathrm{~s}$.

催化剂的 BET 比表面积在美国麦克公司生产的 Tristar 3000 型多功能吸附仪上采用 $\mathrm{N}_{2}$ 吸附法在液氮 温度下测得. 测试前样品在 $200^{\circ} \mathrm{C}$ 进行真空处理.

采用德国 NETZSCH 公司生产的 TG209F1 型同 
步热分析仪进行热重和微分热重(TG-DTG)分析, 以 空气作载气, 空气流速为 $30 \mathrm{~mL} \cdot \mathrm{min}^{-1}$, 催化剂用量 约 $10 \mathrm{mg}$ 左右, 以 $10^{\circ} \mathrm{C} \cdot \mathrm{min}^{-1}$ 的速率升温至 $750^{\circ} \mathrm{C}$.

采用 $\mathrm{N}_{2} \mathrm{O}$ 化学反应吸附法测定经还原后催化剂 中金属铜的比表面积和分散度, $\mathrm{N}_{2} \mathrm{O}$ 化学吸附在美 国 Micromeritics 公司的 AutoChem II 2920 型化学吸 附仪上进行..$^{15}$ 首先, 将 $100 \mathrm{mg}$ 催化剂在 $300{ }^{\circ} \mathrm{C} 、 5 \%$ (体积分数) $\mathrm{H}_{2} / \mathrm{Ar}$ 的气氛下还原 $2 \mathrm{~h}$. 然后用 $\mathrm{Ar}$ 吹扫 并冷却至 $65{ }^{\circ} \mathrm{C}$, 再切换到 $10 \%$ (体积分数) $\mathrm{N}_{2} \mathrm{O} / \mathrm{N}_{2}$ 进 行化学吸附 $1 \mathrm{~h}$, 使得金属 $\mathrm{Cu}$ 完全被氧化为 $\mathrm{Cu}_{2} \mathrm{O}$. 然后用 $\mathrm{Ar}$ 将 $\mathrm{N}_{2} \mathrm{O}$ 吹扫干净并冷却到室温, 在 $300{ }^{\circ} \mathrm{C}$ 的温度下, 采用脉冲纯 $\mathrm{H}_{2}$ 进行还原, 并记录此时的 脉冲 $\mathrm{H}_{2}$ 消耗量.

氢气程序升温还原 $\left(\mathrm{H}_{2}-\mathrm{TPR}\right)$ 在自制的装置上进 行测定. 将 $50 \mathrm{mg}$ 样品放入 $\mathrm{U}$ 型石英管中, 先在 $\mathrm{Ar}$ 吹扫下升温至 $150^{\circ} \mathrm{C}$ 并恒温 $1 \mathrm{~h}$, 以除去吸附在样品 表面的气体杂质. 待温度降至室温后, 将气体切换 成流速为 $30 \mathrm{~mL} \cdot \mathrm{min}^{-1}$ 的 $5 \%$ (体积分数) $\mathrm{H}_{2} / \mathrm{Ar}$ 混合 气, 然后以 $5^{\circ} \mathrm{C} \cdot \mathrm{min}^{-1}$ 的速率升温至 $600{ }^{\circ} \mathrm{C}$, 采用热 导检测器 $(\mathrm{TCD})$ 记录 $\mathrm{H}_{2}$ 消耗的情况.

催化剂的 $\mathrm{H}_{2}$ 程序升温脱附 $\left(\mathrm{H}_{2}-\mathrm{TPD}\right)$ 分析在自 制的脱附装置上进行. 将 $200 \mathrm{mg}$ 样品放入 $\mathrm{U}$ 型石英 管中, 首先用流速为 $30 \mathrm{~mL} \cdot \mathrm{min}^{-1}$ 的 $\mathrm{H}_{2}$ 在 $300{ }^{\circ} \mathrm{C}$ 的 温度下还原 $2 \mathrm{~h}$. 还原后降温至 $50^{\circ} \mathrm{C}$, 并在 $50{ }^{\circ} \mathrm{C}$ 下 进行 $\mathrm{H}_{2}$ 吸附 $1 \mathrm{~h}$, 然后采用流速为 $40 \mathrm{~mL} \cdot \mathrm{min}^{-1}$ 的 $\mathrm{Ar}$ 进行吹扫, 直至平衡. 升温速率为 $10^{\circ} \mathrm{C} \cdot \mathrm{min}^{-1}$, 升温 至 $750{ }^{\circ} \mathrm{C}$. 采用 $\mathrm{TCD}$ 记录 $\mathrm{H}_{2}$ 脱附曲线, 另外, 采用 $\mathrm{H}_{2}$ 脉冲定量测定气体脱附量.

催化剂的 $\mathrm{CO}_{2}$ 程序升温脱附分析 $\left(\mathrm{CO}_{2}-\mathrm{TPD}\right)$ 按 照 $\mathrm{H}_{2}$-TPD 的分析过程, 不同的是该过程采用 AME$\mathrm{TEK}$ 质谱记录 $\mathrm{CO}_{2}$ 脱附曲线 $(m / z=44)$, 采用 $\mathrm{CO}_{2}$ 脉冲 定量测定气体脱附量.

\section{3 催化剂的评价}

催化剂的活性测试在加压连续流动的固定床 不锈钢反应器中进行. 反应前催化剂先在 $\mathrm{H}_{2}$ 中于 $300{ }^{\circ} \mathrm{C}$ 还原 $8 \mathrm{~h}$. 然后冷却到反应温度, 将气体切换 至原料气 $\left(\mathrm{CO}_{2}: \mathrm{H}_{2}\right.$ 摩尔比为 $\left.1: 3\right)$. 反应条件为: $T=$ $250{ }^{\circ} \mathrm{C}, p=5.0 \mathrm{MPa}$, 气体空速 $(\mathrm{GHSV})=12000 \mathrm{~mL} \cdot \mathrm{g}^{-1}$. $\mathrm{h}^{-1}$, 反应产物用上海海欣气相色谱仪进行检测, TDX-01 填充柱和 TCD 用于 $\mathrm{H}_{2} 、 \mathrm{CO}_{2} 、 \mathrm{CO}$ 和 $\mathrm{CH}_{4}$ 的分 离、检测, Porapak Q 柱和氢火焰离子化检测器(FID) 用于液相的水和甲醇等物质的分离和检测. 根据所 测定的反应尾气中各组分的含量, 以碳原子的摩尔
数计算 $\mathrm{CO}_{2}$ 的转化率和产物中各物质的选择性.

\section{3 结果与讨论}

\section{1 催化剂的织构和结构性能}

图 1 为各种前驱体材料的 XRD 谱图. 所有的前 驱体材料皆具有类水滑石结构的特征峰, 没有观察 到其它晶相的衍射峰. 然而, HT-AZr、HT-AY 和 HTAZY 样品衍射峰的强度比 HT-A 弱很多, 说明 $\mathrm{Zr}^{4+}$ 和 $\mathrm{Y}^{3+}$ 离子的引入使得类水滑石晶型规整度下降. 这可 能是大半径的 $\mathrm{Zr}^{4+}$ (离子半径为 $0.072 \mathrm{~nm}$ ) 和 $\mathrm{Y}^{3+}$ (离子 半径为 $0.090 \mathrm{~nm}$ ) 取代 $\mathrm{Al}^{3+}$ (离子半径为 $0.053 \mathrm{~nm}$ ) ${ }^{16}$ 使类水滑石层板发生较大程度的扭曲, 进而导致结 晶度降低.

基于类水滑石化合物属于六方晶系, 计算了晶 胞参数 $a$ 和 $c$, 结果列于表 1 中. 从表 1 可以看到, 晶 胞参数 $a$ 和 $c$ 均随 $\mathrm{Zr}$ 和 $\mathrm{Y}$ 的引入而增加. 晶胞参数 $a\left(=2 d_{(110)}\right)$ 取决于层板上金属阳离子的平均半径以 及 $(001)$ 晶面上的金属离子的分布密度. ${ }^{7}$ 参数 $a$ 的增 加是由于在 $\mathrm{HTl}$ 层板中大半径的 $\mathrm{Zr}^{4+}$ 和 $\mathrm{Y}^{3+}$ 离子同晶 取代了 $\mathrm{Al}^{3+}$. 晶胞参数 $c\left(=d_{(003)}+2 d_{(006)}+3 d_{(009)}\right)$ 与类水 滑石层间距 $(d)$ 密切相关, 由层板厚度、层板的电荷 密度、层间阴离子的大小及层间水分子数量所决 定. ${ }^{18,19}$ 小半径的阳离子 $\left(\mathrm{Al}^{3+}\right)$ 被大半径的阳离子 $\left(\mathrm{Zr}^{4+}\right.$ 和 $\mathrm{Y}^{3+}$ )取代会增加类水滑石层板的厚度. 另外, HT$\mathrm{AZ}$ 样品的晶胞参数 $c$ 比 HT-AY 的小, 这是因为四价 阳离子 $\mathrm{Zr}^{4+}$ 的引入使层板正电荷密度增加, 增强了 层板与层间阴离子间的库仑作用力. ${ }^{15}$

图 2 为 $500{ }^{\circ} \mathrm{C}$ 焙烧后样品的 XRD 谱图. 很明 显, 在谱图中已经观察不到类水滑石化合物的特征 峰, 所有的样品均可以观察到一些宽化的衍射峰,

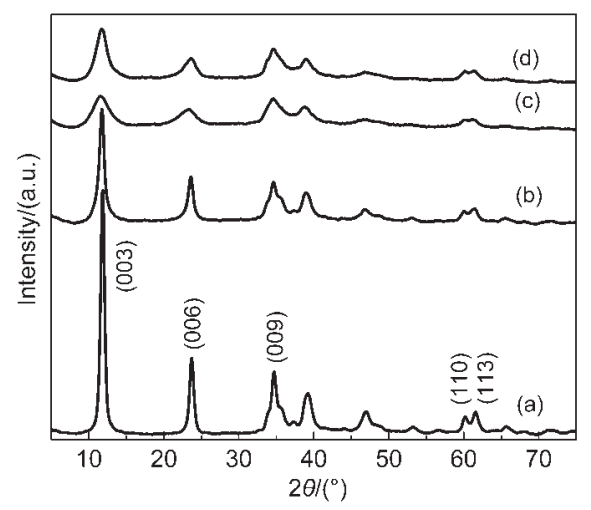

图 1 未焙烧的 $\mathrm{Cu} / \mathrm{Zn} / \mathrm{Al} /(\mathrm{Zr}) /(\mathrm{Y})$ 前驱体材料的 XRD 谱图

Fig.1 XRD patterns of the uncalcined $\mathrm{Cu} / \mathrm{Zn} / \mathrm{Al} /$ $(\mathrm{Zr}) /(\mathrm{Y})$ precursors

(a) HT-A; (b) HT-AZ; (c) HT-AY; (d) HT-AZY 
表 1 前驱体样品的结构参数、组成和失重(发生在第二和第三热分解阶段)

Table 1 Structure, composition, and weight loss (occurring in the second and third steps of thermal decomposition) of the precursors

\begin{tabular}{lcccc}
\hline Sample & Atomic ratio of Cu:Zn:Al:Zr:Y ${ }^{a}$ & $a / \mathrm{nm}$ & $c / \mathrm{nm}$ & $w($ second/third step)/\% \\
\hline HT-A & $2: 1: 1: 0: 0$ & 0.3078 & 2.273 & $11.0 / 5.5$ \\
HT-AZ & $2: 1: 0.8: 0.2: 0$ & 0.3084 & 2.276 & $13.0 / 4.5$ \\
HT-AY & $2: 1: 0.8: 0: 0.2$ & 0.3083 & 2.309 & $16.0 / 4.0$ \\
HT-AZY & $2: 1: 0.8: 0.1: 0.1$ & 0.3084 & 2.294 & $16.0 / 4.0$ \\
\hline
\end{tabular}

${ }^{a}$ the nominal atomic ratio in the synthesis mixture

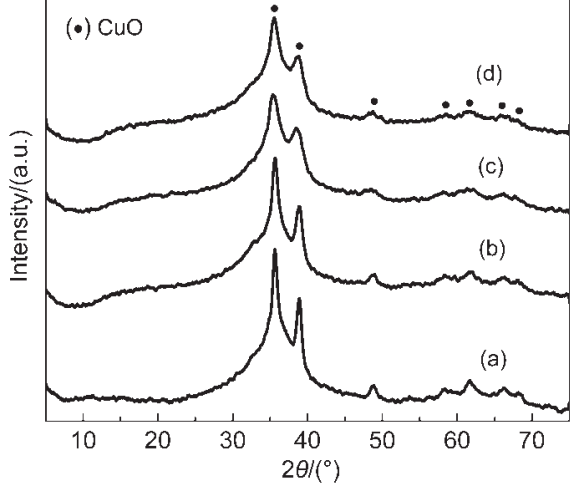

图 2 焙烧后 $\mathrm{Cu} / \mathrm{Zn} / \mathrm{Al} /(\mathrm{Zr}) /(\mathrm{Y})$ 催化剂的 XRD 谱图

Fig.2 XRD patterns of the calcined $\mathrm{Cu} / \mathrm{Zn} / \mathrm{Al}$ / $(\mathrm{Zr}) /(\mathrm{Y})$ catalysts

(a) CHT-A; (b) CHT-AZ; (c) CHT-AY; (d) CHT-AZY

这归属于结晶度较差的 $\mathrm{CuO}$ 晶相, 并且这些衍射峰 的强度随助剂的引入而降低, $\mathrm{Y}$ 的引入使得 $\mathrm{CuO}$ 衍 射峰强度降低得更加明显. 然而, 在所有样品的 XRD 谱图中, 均没有观察到其它金属氧化物的衍射 峰, 说明它们以无定形或微晶状态存在. ${ }^{10,20}$

图 3 为不同助剂修饰的类水滑石化合物的 TGDTG 曲线. 通常类水滑石材料的热分解包含三个阶 段. 第一失重阶段发生在 $50-150{ }^{\circ} \mathrm{C}$ 之间, 对应于类 水滑石化合物失去物理吸附和层间的水分子. 从图 中可以看到, 助剂的添加使得水分子脱除发生在更 低温度处, 这是由于 $\mathrm{Zr}^{4+}$ 和 $\mathrm{Y}^{3+}$ 离子的引入使得水分 子和层间阴离子的氢键强度降低. ${ }^{919}$ 第二阶段的失 重发生在 150-550 ${ }^{\circ} \mathrm{C}$ 之间, 对应于层板上羟基基团 的脱水和层间碳酸根离子的脱除, 这导致了层状结 构的坍塌. ${ }^{21,22}$ 如图 3 所示, $\mathrm{Zr}^{4+}$ 和 $\mathrm{Y}^{3+}$ 离子引入类水滑 石结构中使得该阶段的失重温度向低温位移, 这说 明类水滑石结构的稳定性降低了. 从表 1 中可以看 到, 助剂的引入使得第二阶段失重量明显增加, 且 HT-AY 和 HT-AZY 样品的失重量高于 HT-AZ, 这说 明 $\mathrm{Y}$ 的引入使得类水滑石结构分解产生的 $\mathrm{H}_{2} \mathrm{O}$ 和 $\mathrm{CO}_{2}$ 的含量更高. 第三步失重发生在 $500-750{ }^{\circ} \mathrm{C}$ 之 间, 这一阶段的失重归结为稳定的含铜碳酸氧盐的 分解, 该碳酸氧盐物种是在类水滑石结构热分解的

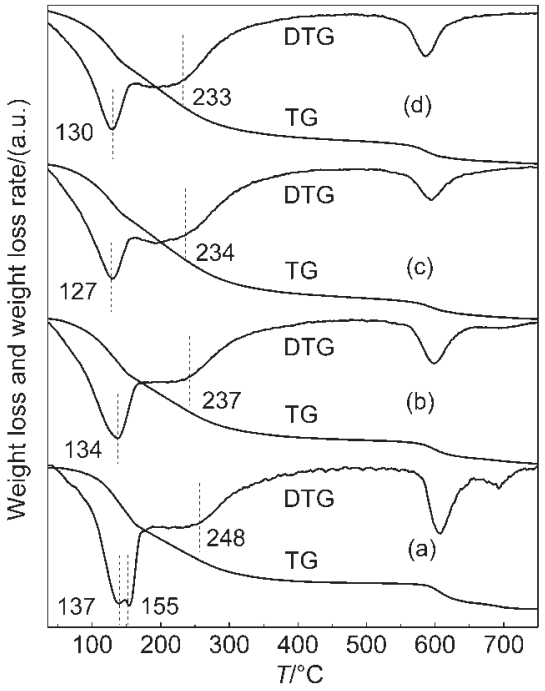

图 3 前驱体材料在空气中的热重和微分热重 (TG-DTG)曲线

Fig.3 Thermogravimetry and differential thermogravimetry (TG-DTG) profiles of precursors in air (a) CHT-A; (b) CHT-AZ; (c) CHT-AY; (d) CHT-AZY

前两个阶段形成的. ${ }^{23,24}$ 表 1 中可以看到第三阶段失 重量即含铜碳酸氧盐的含量依次降低: HT-A>HT$A Z>H T-A Y \approx H T-A Z Y$, 这些含铜物种具有更高的热 稳定性, 且不易还原从而会降低催化性能. ${ }^{20,24,25}$

焙烧后样品的比表面积和孔体积列于表 2 中. $\mathrm{Zr}$ 和 $\mathrm{Y}$ 的引入可以增加 BET 比表面积 $\left(S_{\mathrm{BET}}\right)$ 和孔体 积 $\left(V_{\mathrm{p}}\right)$, 并且 $\mathrm{Y}$ 的引入对两者的增加更为显著. 根据 $\mathrm{TG}$ 分析, 该现象可能与前驱体材料焙烧过程中 $\mathrm{H}_{2} \mathrm{O}$

表 $2 \mathrm{Cu} / \mathrm{Zn} / \mathrm{Al} /(\mathrm{Zr}) /(\mathrm{Y})$ 催化剂的物理化学性质

Table 2 Physicochemical properties of the $\mathrm{Cu} / \mathrm{Zn} / \mathrm{Al} /(\mathbf{Z r}) /(\mathbf{Y})$ catalysts

\begin{tabular}{lcccrr}
\hline Sample & $S_{\mathrm{BET}} /\left(\mathrm{m}^{2} \cdot \mathrm{g}^{-1}\right)$ & $V_{\mathrm{p}} /\left(\mathrm{cm}^{3} \cdot \mathrm{g}^{-1}\right)$ & $S_{\mathrm{Cu}} /\left(\mathrm{m}^{2} \cdot \mathrm{g}^{-1}\right)$ & $D_{\mathrm{Cu}} / \%$ & $d_{\mathrm{Cu}} / \mathrm{nm}$ \\
\hline CHT-A & 39 & 0.20 & 14.4 & 5.57 & 18.7 \\
CHT-AZ & 67 & 0.30 & 27.6 & 10.82 & 9.6 \\
CHT-AY & 108 & 0.76 & 33.8 & 13.17 & 7.9 \\
CHT-AZY & 105 & 0.68 & 34.1 & 13.50 & 7.7 \\
\hline
\end{tabular}

$S_{\text {BET: }}$ specific surface area of catalyst; $V_{\mathrm{p}}$ : pore volume of catalyst;

$S_{\text {Cu: }}$ the specific surface area of metal Cu determined by $\mathrm{N}_{2} \mathrm{O}$ dissociative adsorption; $D_{\mathrm{Cu}}$ : the dispersion of $\mathrm{Cu}$; $d_{\mathrm{cu}}$ : the average $\mathrm{Cu}$ particle size 
和 $\mathrm{CO}_{2}$ 的生成量有关. 采用 $\mathrm{N}_{2} \mathrm{O}$ 反应吸附法测定了 还原后催化剂中金属铜的比表面积 $\left(S_{\mathrm{Cu}}\right)$ 、铜的分散 度 $\left(D_{\mathrm{Cu}}\right)$ 和铜的平均粒径 $\left(d_{\mathrm{Cu}}\right)$. 如表 2 所示, $\mathrm{Zr}$ 和 $\mathrm{Y}$ 的 引入可以大大降低 $d_{\mathrm{Cu}}$, 同时, 显著提高 $S_{\mathrm{Cu}}$ 和 $D_{\mathrm{Cu}}$. 另 外, $S_{\mathrm{Cu}}$ 和 $D_{\mathrm{Cu}}$ 均按以下顺序依次增加: CHT-A $<\mathrm{CHT}$ $\mathrm{AZ}<\mathrm{CHT}-\mathrm{AY}<\mathrm{CHT}-\mathrm{AZY}$, 而 $d_{\mathrm{Cu}}$ 则按该顺序依次降 低, 这说明 $\mathrm{Zr}$ 和 $\mathrm{Y}$ 助剂共同修饰的 $\mathrm{Cu} / \mathrm{Zn} / \mathrm{Al}$ 催化剂 具有最高的 $S_{\mathrm{Cu}}$ 和 $D_{\mathrm{Cu}}$.

\section{2 催化剂的还原性能}

为了研究 $\mathrm{Cu} / \mathrm{Zn} / \mathrm{Al} /(\mathrm{Zr}) /(\mathrm{Y})$ 催化剂的还原行 为, 对催化剂进行了 TPR 测试. 如图 4 所示, 在 200$350{ }^{\circ} \mathrm{C}$ 温度范围内, 所有样品的还原曲线呈现一个 宽的 $\mathrm{H}_{2}$ 消耗峰且伴有肩峰存在. 为了更好的对 $\mathrm{TPR}$ 结果进行分析, 可以将该 $\mathrm{H}_{2}$ 消耗峰分为几个 Gaussian 峰. 表 3 列出了还原峰的峰温和每个峰在 总的 TPR还原峰中所占的比例.

$\beta$ 和 $\gamma$ 峰可以归属于两种不同类型的 $\mathrm{CuO}$ 的还原 峰: 在低温被还原的分散 $\mathrm{CuO}(\beta$ 峰 $)$ 和在高温被还原 的体相 $\mathrm{CuO}$ ( $\gamma$ 峰). ${ }^{26,27}$ 对于 $\mathrm{Cu} / \mathrm{Zn} / \mathrm{Al} / \mathrm{Zr} / \mathrm{Y}$ 催化剂, 在 更低温度处还观察到一个还原峰( $\alpha$ 峰), 它可以归属 于高分散 $\mathrm{CuO}$ 的还原, 这说明 $\mathrm{Zr}$ 和 $\mathrm{Y}$ 的共同引入可

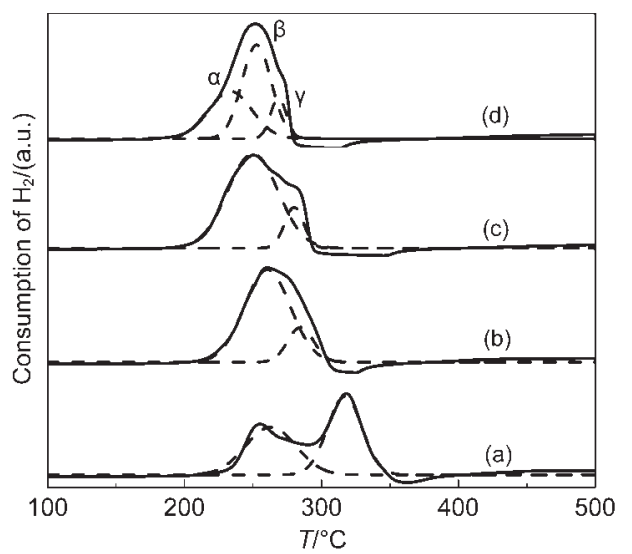

图 $4 \mathrm{Cu} / \mathrm{Zn} / \mathrm{Al} /(\mathrm{Zr}) /(\mathrm{Y})$ 催化剂的 $\mathrm{H}_{2}$-TPR 曲线

Fig.4 $\mathrm{H}_{2}$-TPR profiles of the $\mathrm{Cu} / \mathrm{Zn} / \mathrm{Al} /(\mathrm{Zr}) /(\mathrm{Y})$ catalysts (a) CHT-A; (b) CHT-AZ; (c) CHT-AY; (d) CHT-AZY

表 $3 \mathrm{Cu} / \mathrm{Zn} / \mathrm{Al} /(\mathrm{Zr}) /(\mathrm{Y})$ 催化剂的还原峰温度及每个 还原峰占总 TPR 还原峰的比例

Table 3 Temperatures of reduction peaks and their contributions to the TPR pattern over $\mathrm{Cu} / \mathrm{Zn} / \mathrm{Al} /(\mathrm{Zr}) /(\mathrm{Y})$

\begin{tabular}{lccc}
\hline \multirow{2}{*}{ Sample } & \multicolumn{3}{c}{ TPR peak position temperature $/{ }^{\circ} \mathrm{C}^{\mathrm{a}}$} \\
\cline { 2 - 4 } & peak $\alpha$ & peak $\beta$ & peak $\gamma$ \\
\hline CHT-A & - & $263(48.5)$ & $317(51.5)$ \\
CHT-AZ & - & $261(82.5)$ & $285(17.5)$ \\
CHT-AY & - & $251(85.9)$ & $281(14.1)$ \\
CHT-AZY & $234(37.4)$ & $253(50.9)$ & $269(11.7)$ \\
\hline
\end{tabular}

${ }^{a}$ Values in parentheses are the contribution (\%) of each species.
以使一部分 $\mathrm{CuO}$ 更容易还原. 如表 3 所示, $\alpha$ 和 $\beta$ 两峰 之和所占的比例按如下顺序依次增加: CHT-A $<$ CHT-AZ $<$ CHT-AY $<$ CHT-AZY, CHT-AZY 样品的 $\alpha$ 和 $\beta$ 峰之和所占的比例最大, 为 $88.9 \%$. 这说明分散的、 易还原的 $\mathrm{CuO}$ 的数量从 CHT-A 到 CHT-AZY 逐渐增 加, 这与 $\mathrm{N}_{2} \mathrm{O}$ 化学反应吸附得到的 $D_{\mathrm{Cu}}$ 结果是一致 的. 另外, 将 $\mathrm{Zr}$ 和 $\mathrm{Y}$ 助剂引入 $\mathrm{Cu} / \mathrm{Zn} / \mathrm{Al}$ 催化剂后, $\beta$ 和 $\gamma$ 峰的温度向低温移动, 且 $\gamma$ 峰温度的降低更加显著.

\section{$3.3 \mathrm{H}_{2}$-TPD 分析}

图 5 为还原后催化剂的 $\mathrm{H}_{2}-\mathrm{TPD}$ 曲线. 所有样品 在一个宽的温度范围内 $\left(50-550^{\circ} \mathrm{C}\right)$, 都能观察到 $\mathrm{H}_{2}$ 脱附曲线, 这表明在还原后催化剂的表面存在不同 的氢吸附物种. 在 $140{ }^{\circ} \mathrm{C}$ 左右的低温脱附峰( $\alpha$ 峰)代 表吸附在金属 $\mathrm{Cu}$ 表面的原子 $\mathrm{H}$ 的脱附, ${ }^{28-31}$ 而在 $390{ }^{\circ} \mathrm{C}$ 左右的高温宽峰则对应体相 $\mathrm{Cu}$ 颗粒 ${ }^{28,30,31}$ 或 $\mathrm{ZnO}$ 表面 ${ }^{3}$ 强吸附的氢气脱附. $\mathrm{Al}_{2} \mathrm{O}_{3}$ 不吸附 $\mathrm{H}_{2}$, 因而 不影响 $\mathrm{H}_{2}$ 在其它位点的吸附. ${ }^{31}$ 脱附峰温度和脱附 量列于表 4 中, 从 $\alpha$ 峰的脱附数据可以看到, 随着 $\mathrm{Zr}$ 和 $\mathrm{Y}$ 的引入, $\mathrm{H}_{2}$ 的脱附量明显增加并按如下顺序依 次增加: CHT-A $<$ CHT-AZ $<$ CHT-AY $<$ CHT-AZY, CHT$\mathrm{AZY}$ 样品上的脱附量最大, 为 $114.5 \mu \mathrm{mol} \cdot \mathrm{g}^{-1}$, 这与 $S_{\mathrm{Cu}}$ 的变化(表 1)是一致的. 然而, 高温 $\mathrm{H}_{2}$ 脱附量按如 下顺序依次降低: CHT-A $>$ CHT-AZ $>$ CHT-AY $>$ CHT$\mathrm{AZY}$, 根据 TPR 结果, 这可以部分归结为体相 $\mathrm{Cu}$ 数 量的减少. 另外, $\mathrm{ZnO}$ 表面的 $\mathrm{H}_{2}$ 脱附量也可能按相 同趋势降低.

\section{4 催化剂的表面碱性}

图 6 是还原后催化剂的 $\mathrm{CO}_{2}-\mathrm{TPD}$ 曲线. 三个 Gaussian 峰分别代表弱碱位( $\alpha$ 峰)、中等碱位( $\beta$ 峰)和

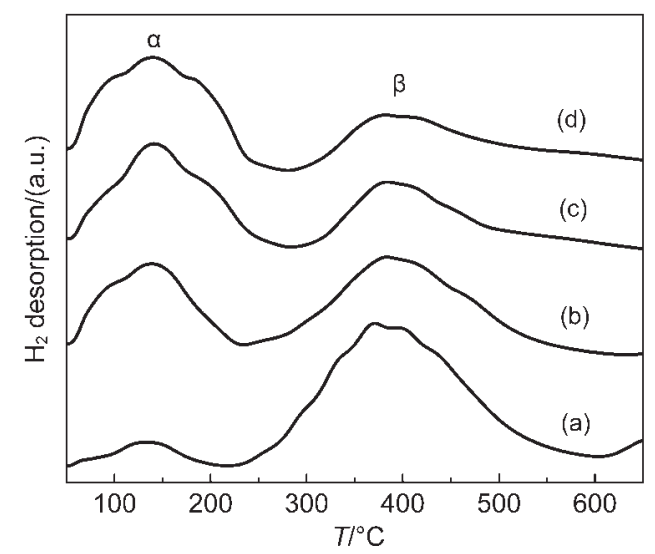

图 5 还原后 $\mathrm{Cu} / \mathrm{Zn} / \mathrm{Al} /(\mathrm{Zr}) /(\mathrm{Y})$ 催化剂的 $\mathrm{H}_{2}-\mathrm{TPD}$ 曲线

Fig.5 $\mathrm{H}_{2}$-TPD profiles of the pre-reduced $\mathrm{Cu} / \mathrm{Zn} /$ $\mathbf{A l} /(\mathbf{Z r}) /(\mathbf{Y})$ catalysts

(a) CHT-A; (b) CHT-AZ; (c) CHT-AY; (d) CHT-AZY 
表4 还原后 $\mathrm{Cu} / \mathrm{Zn} / \mathrm{Al} /(\mathrm{Zr}) /(\mathrm{Y})$ 催化剂的脱附峰温度和 $\mathbf{H}_{2}$ 脱附量

Table 4 The maximum temperature and amount of $\mathbf{H}_{2}$ desorption over pre-reduced $\mathrm{Cu} / \mathrm{Zn} / \mathrm{Al} /(\mathrm{Zr}) /(\mathrm{Y})$ catalysts

\begin{tabular}{|c|c|c|c|c|}
\hline \multirow{3}{*}{ Sample } & \multicolumn{2}{|r|}{ Peak $\alpha$} & \multicolumn{2}{|r|}{ Peak $\beta$} \\
\hline & \multirow{2}{*}{$T /{ }^{\circ} \mathrm{C}$} & \multirow{2}{*}{$\frac{\mathrm{H}_{2} \text { desorption }}{\left(\mu \mathrm{mol} \cdot \mathrm{g}^{-1}\right)}$} & \multirow{2}{*}{$T /{ }^{\circ} \mathrm{C}$} & $\mathrm{H}_{2}$ desorption \\
\hline & & & & $\left(\mu \mathrm{mol} \cdot \mathrm{g}^{-1}\right)$ \\
\hline CHT-A & 133 & 20.4 & 384 & 392.8 \\
\hline CHT-AZ & 139 & 86.2 & 386 & 215.8 \\
\hline CHT-AY & 142 & 111.9 & 391 & 102.5 \\
\hline CHT-AZY & 140 & 114.5 & 394 & 86.7 \\
\hline
\end{tabular}

强碱位( $\gamma$ 峰). 弱碱位是由表面 $\mathrm{OH}^{-}$基团产生的, 中 等碱位是由金属-氧离子对产生的, 而强碱位则由配

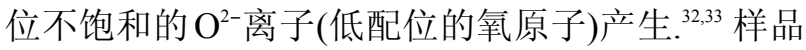
的碱位数目以及各碱位数目占总碱位数目的比例 列于表 5 中. 助剂的加入使得总碱位数目增多, 并且 各催化剂按如下顺序依次增加: CHT-A $<$ CHT-AZ $<$ CHT-AZY $<$ CHT-AY. 对单个的 $\alpha 、 \beta$ 及 $\gamma$ 碱位而言, 碱 位数目也呈现类似的增加趋势. 比表面积增加会使 得暴露的碱性位增多, 这可以解释碱位数目从 CHTA 到 CHT-AY 是依次增加的. 然而, 各碱位比例的变 化趋势有所不同, 强碱位数目占总碱位数目的比例 按如下顺序依次增加: CHT- A $<$ CHT- AY $<$ CHTAZY $<$ CHT-AZ, CHT-AZ样品的强碱位比例最大, 为 $21.9 \%$, 其次是 CHT-AZY 样品, 这是因为四价 $\mathrm{Zr}^{4+}$ 进 入类水滑石结构后使得层板净电荷增加, 从而使配

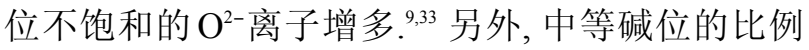
则呈相反的变化趋势.

\section{5 催化剂的催化性能}

$\mathrm{CO}_{2}$ 加氢合成甲醇的主要产物是甲醇、 $\mathrm{CO}$ 和

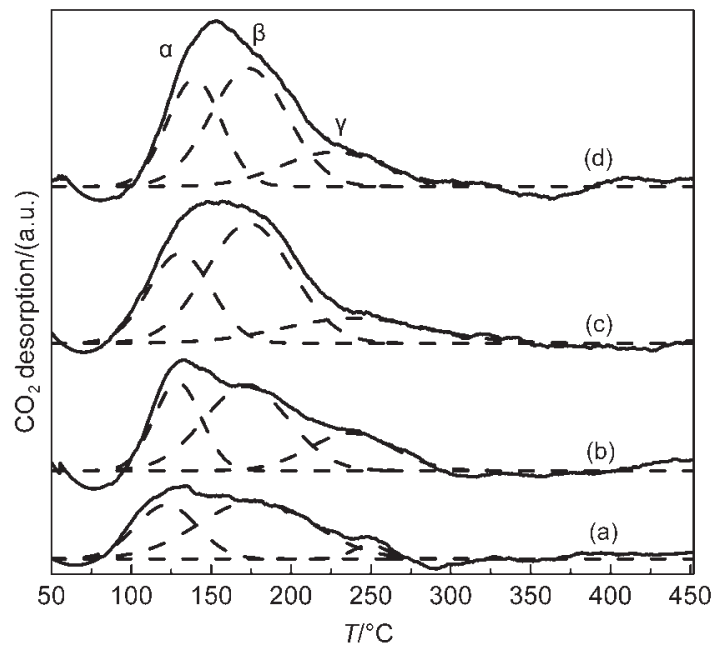

图 6 还原后 $\mathrm{Cu} / \mathrm{Zn} / \mathrm{Al} /(\mathrm{Zr}) /(\mathrm{Y})$ 催化剂的 $\mathrm{CO}_{2}-\mathrm{TPD}$ 曲线

Fig.6 $\mathrm{CO}_{2}$-TPD profiles of pre-reduced $\mathrm{Cu} / \mathrm{Zn} / \mathrm{Al} /$ $(\mathrm{Zr}) /(\mathrm{Y})$ catalysts

(a) CHT-A; (b) CHT-AZ; (c) CHT-AY; (d) CHT-AZY

水, 极少量的 $\mathrm{CH}_{4}$ 和高级烃类也能检测到. 表 6 列出 了不同助剂修饰的 $\mathrm{Cu} / \mathrm{Zn} / \mathrm{Al}$ 催化剂的催化性能. $\mathrm{CO}_{2}$ 转化率随 $\mathrm{Zr}$ 和 $\mathrm{Y}$ 助剂的引入而增加, 并且各样 品的 $\mathrm{CO}_{2}$ 转化率增加顺序依次为: CHT- $<<$ CHT$\mathrm{AZ}<\mathrm{CHT}-\mathrm{AY}<\mathrm{CHT}-\mathrm{AZY}$, 这与铜的比表面积 $\left(S_{\mathrm{Cu}}\right)$ 的 变化是一致的(如图 7 所示). 对于 $\mathrm{Cu} / \mathrm{Zn} / \mathrm{Al}$ 或 $\mathrm{Cu} / \mathrm{Zn} /$ $\mathrm{Al} / \mathrm{Zr}$ 催化剂, $\mathrm{CO}_{2}$ 加氢合成甲醇的双活性中心的反 应机理被广泛认可, $\mathrm{H}_{2}$ 的吸附解离在 $\mathrm{Cu}$ 位点上进 行, 而 $\mathrm{CO}_{2}$ 的吸附则发生在 $\mathrm{ZnO}$ 或 $\mathrm{ZrO}_{2}$ 等碱性位点 上, $\mathrm{Cu}$ 位点上的原子 $\mathrm{H}$ 通过溢流作用到达碱性位点 表面, 对含碳物种进行逐步加氢形成甲酸盐、表面 键合甲醛、甲醇盐, 最后形成甲醇. $728,29,34$ 一些研究者 还发现, 在 $\mathrm{CO}_{2}$ 加氢反应过程中, $\mathrm{CO}_{2}$ 的吸附量是充

表 5 还原后 $\mathrm{Cu} / \mathrm{Zn} / \mathrm{Al} /(\mathrm{Zr}) /(\mathrm{Y})$ 催化剂的碱位数目以及各碱位所占的比例

Table 5 Basicity and distribution of basic sites over reduced $\mathrm{Cu} / \mathrm{Zn} / \mathrm{Al} /(\mathrm{Zr}) /(\mathrm{Y})$ catalysts

\begin{tabular}{lcccc}
\hline \multirow{2}{*}{ Sample } & \multirow{2}{*}{ Amount of total basic sites $/\left(\mu \mathrm{mol} \cdot \mathrm{g}^{-1}\right)$} & \multicolumn{3}{c}{ Amount of basic sites $/\left(\mu \mathrm{mol} \cdot \mathrm{g}^{-1}\right)^{\mathrm{a}}$} \\
\cline { 3 - 5 } CHT-A & 121.9 & site $\alpha$ & site $\beta$ & $6.4(5.3)$ \\
CHT-AZ & 194.6 & $54.5(36.5)$ & $71.1(58.2)$ & $42.5(21.9)$ \\
CHT-AY & 263.0 & $76.0(28.8)$ & $96.0(49.3)$ & $48.5(18.4)$ \\
CHT-AZY & 248.6 & $77.6(31.2)$ & $138.5(52.7)$ & $42.7(49.4)$ \\
\hline
\end{tabular}

${ }^{a}$ values in the parentheses: contribution (\%) of the single basic site to the number of total basic sites

表 $6 \mathrm{Cu} / \mathrm{Zn} / \mathrm{Al} /(\mathrm{Zr}) /(\mathrm{Y})$ 催化剂上 $\mathrm{CO}_{2}$ 加氢合成甲醇的催化性能

Table 6 Catalytic performance for methanol synthesis from $\mathrm{CO}_{2}$ hydrogenation over $\mathrm{Cu} / \mathrm{Zn} / \mathrm{Al} /(\mathrm{Zr}) /(\mathrm{Y})$ catalysts

\begin{tabular}{lccccccc}
\hline \multirow{2}{*}{ Sample } & \multirow{2}{*}{$\mathrm{CO}_{2}$ conversion/\% } & \multirow{2}{*}{$10^{-4} \mathrm{TOF} / \mathrm{s}^{-1}$} & \multicolumn{3}{c}{ Selectivity $/ \%$} & \multirow{2}{*}{$\mathrm{CH}_{3} \mathrm{OH}$ yield $/\left(\mathrm{g} \cdot \mathrm{g}^{-1} \cdot \mathrm{h}^{-1}\right)$} \\
\cline { 5 - 6 } & & 1.827 & 39.7 & 0.6 & 59.7 & 0.34 \\
CHT-A & 19.7 & 1.256 & 50.7 & 0.6 & 48.7 & 0.46 \\
CHT-AZ & 22.1 & 1.088 & 47.9 & 0.6 & 51.5 & 0.48 \\
CHT-AY & 24.9 & 1.035 & 49.8 & 0.6 & 49.6 & 0.52 \\
CHT-AZY & 26.0 & $\mathrm{CH}_{4}$ & $\mathrm{CO}$ & \\
\hline
\end{tabular}

reaction conditions: $T=250^{\circ} \mathrm{C}, p=5.0 \mathrm{MPa}$, GHSV (gas hourly space velocity) $=12000 \mathrm{~mL} \cdot \mathrm{g}^{-1} \cdot \mathrm{h}^{-1}, n\left(\mathrm{H}_{2}\right): n\left(\mathrm{CO}_{2}\right)=3: 1 ;$ TOF: turnover frequency 


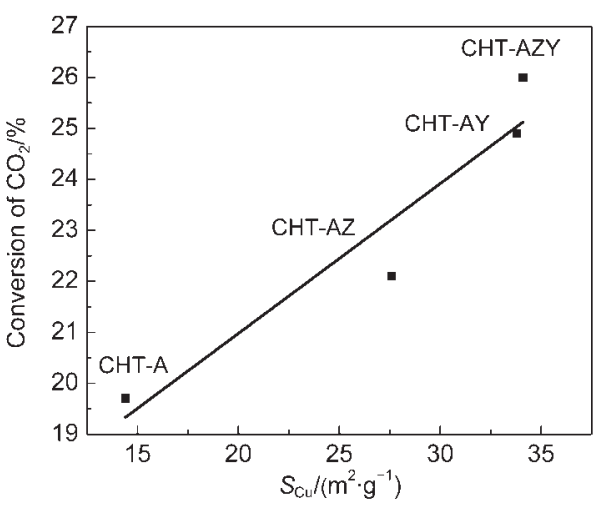

图 $7 \mathrm{CO}_{2}$ 转化率与 $\mathrm{Cu}$ 的比表面积之间的关系

Fig.7 Relationship between the conversion of $\mathrm{CO}_{2}$ and the $\mathrm{Cu}$ specific surface area

reaction conditions: $T=250{ }^{\circ} \mathrm{C}, p=5.0 \mathrm{MPa}, \mathrm{GHSV}=12000 \mathrm{~mL} \cdot \mathrm{g}^{-1} \cdot \mathrm{h}^{-1}$, $n\left(\mathrm{H}_{2}\right): n\left(\mathrm{CO}_{2}\right)=3: 1$

足的, 而形成的原子 $\mathrm{H}$ 是不足量的. 鉴于此, $\mathrm{CO}_{2}$ 转 化率和 $S_{\mathrm{Cu}}$ 之间的关系可以解释如下: $S_{\mathrm{Cu}}$ 的增加会导 致吸附解离的原子 $\mathrm{H}$ 增加(这一点已经得到 $\mathrm{H}_{2}-\mathrm{TPD}$ 实验结果的支持), 因而有更多的原子 $\mathrm{H}$ 通过溢流到 达碱性位点表面对吸附的碳物种进行加氢反应, 从 而提高 $\mathrm{CO}_{2}$ 的转化率. 这也说明 $S_{\mathrm{Cu}}$ 对 $\mathrm{Cu}$ 基催化剂 的催化性能起着重要的作用.

为了对比催化剂表面单位 $\mathrm{Cu}$ 原子的催化效率, 我们还计算了各个催化剂上的频率转换因子 (TOF), 并将结果列于表 6 中. $\mathrm{Zr}$ 和 $\mathrm{Y}$ 助剂的引入使 TOF 降低, CHT-AZY 催化剂的 TOF 最小, 为 $1.035 \times$ $10^{4} \mathrm{~s}^{-1}$. 图 8 揭示了 TOF 与金属铜粒径 $\left(d_{\mathrm{Cu}}\right)$ 之间的关 系. 显然, 随着 $d_{\mathrm{cu}}$ 值的增加, TOF 几乎呈线性增加, 类似的变化已有报道. ${ }^{26,35}$ 这表明 $\mathrm{CO}_{2}$ 加氢合成甲醇 反应是一个结构敏感型反应.

$\mathrm{Zr}$ 和 $\mathrm{Y}$ 助剂的引入对甲醇选择性的提高也十

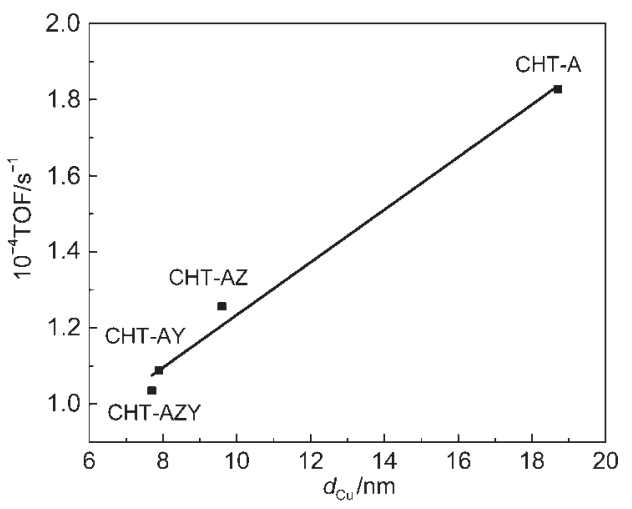

图 $8 \mathrm{TOF}$ 与 $\mathrm{Cu}$ 的粒径之间的关系

Fig.8 Relationship between TOF and $\mathrm{Cu}$ particle size reaction conditions: $T=250^{\circ} \mathrm{C}, p=5.0 \mathrm{MPa}, \mathrm{GHSV}=12000$ $\mathrm{mL} \cdot \mathrm{g}^{-1} \cdot \mathrm{h}^{-1}, n\left(\mathrm{H}_{2}\right): n\left(\mathrm{CO}_{2}\right)=3: 1$

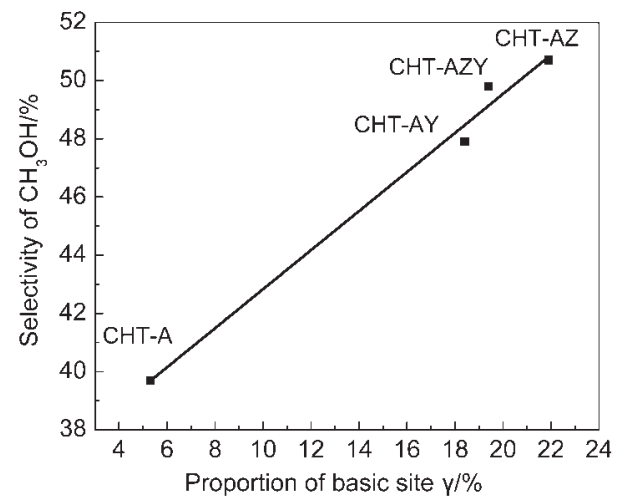

图9甲醇选择性与强碱位 $\gamma$ 比例之间的关系

Fig.9 Relationship between the selectivity of $\mathrm{CH}_{3} \mathrm{OH}$ and the proportion of strongly basic sites $\gamma$ to the total basic sites

reaction conditions: $T=250^{\circ} \mathrm{C}, p=5.0 \mathrm{MPa}, \mathrm{GHSV}=12000$ $\mathrm{mL} \cdot \mathrm{g}^{-1} \cdot \mathrm{h}^{-1}, n\left(\mathrm{H}_{2}\right): n\left(\mathrm{CO}_{2}\right)=3: 1$

分显著, 相对于 CHT-A 催化剂, $\mathrm{Zr}$ 和 $\mathrm{Y}$ 助剂的引入 使得甲醇选择性增加了 $21 \%-28 \%$. 另外, $\mathrm{Zr}$ 修饰的 $\mathrm{Cu} / \mathrm{Zn} / \mathrm{Al}$ 催化剂的甲醇选择性比 $\mathrm{Y}$ 修饰的催化剂 高, 并且 CHT-AZY 的甲醇选择性略低于 CHT-AZ. 根据我们前期研究 ${ }^{9,15}$ 中所提出的 $\mathrm{CO}_{2}$ 加氢合成甲醇 的反应机理, 甲醇选择性与碱位分布有关. 图 9 给出 了甲醇选择性与强碱位 $\gamma$ 比例之间的关系, 从图 9 中 可以看到, 甲醇选择性与强碱位 $\gamma$ 的比例呈线性关 系. 一些研究者认为甲醇和 CO 均通过相同的中间 体形成, 相对于中等碱位, 吸附在强碱位上的中间 物种更倾向于进一步加氢生成甲醇而不是分解形 成 CO, 因此甲醇选择性与强碱位的比例有关. ${ }^{15,29,36}$ 各样品的甲醇收率与 $\mathrm{CO}_{2}$ 转化率的变化顺序相一 致. $\mathrm{Zr}$ 和 $\mathrm{Y}$ 助剂共同修饰的 $\mathrm{Cu} / \mathrm{Zn} / \mathrm{Al}$ 催化剂上的甲 醇收率最大, 达到 $0.52 \mathrm{~g} \cdot \mathrm{g}^{-1} \cdot \mathrm{h}^{-1}$, 其 $\mathrm{CO}_{2}$ 转化率为 $26.0 \%$, 甲醇选择性为 $49.8 \%$.

\section{4 结 论}

采用共沉淀法将少量 $\mathrm{Zr}$ 和 $\mathrm{Y}$ 引入到了 $\mathrm{Cu} / \mathrm{Zn} / \mathrm{Al}$ 类水滑石结构中, 相应的类水滑石前驱体焙烧后得 到 $\mathrm{Cu} / \mathrm{Zn} / \mathrm{Al} 、 \mathrm{Cu} / \mathrm{Zn} / \mathrm{Al} / \mathrm{Zr} 、 \mathrm{Cu} / \mathrm{Zn} / \mathrm{Al} / \mathrm{Y}$ 和 $\mathrm{Cu} / \mathrm{Zn} / \mathrm{Al} / \mathrm{Zr} / \mathrm{Y}$ 四种催化剂. $\mathrm{Zr}$ 和 $Y$ 助剂对 $\mathrm{Cu} / \mathrm{Zn} / \mathrm{Al}$ 催化剂的物理 化学性质和 $\mathrm{CO}_{2}$ 加氢合成甲醇的催化性能有显著影 响. 助剂的引入可以显著提高催化剂的 BET 比表面 积、金属铜的比表面积和分散度以及碱位数目，同 时, 金属铜的粒径大幅降低. 另外, Y 助剂对催化剂 比表面积的提高更明显, 这与前驱体材料焙烧过程 中 $\mathrm{H}_{2} \mathrm{O}$ 和 $\mathrm{CO}_{2}$ 的生成量有关, $\mathrm{Zr}$ 和 $\mathrm{Y}$ 共同修饰的 $\mathrm{Cu} /$ 
$\mathrm{Zn} / \mathrm{Al}$ 催化剂具有最高的铜的比表面积和分散度, 然而, $\mathrm{Zr}$ 修饰的 $\mathrm{Cu} / \mathrm{Zn} / \mathrm{Al}$ 催化剂的强碱位比例最 大. $\mathrm{CO}_{2}$ 转化率取决于铜的比表面积, 并按以下顺序 依次增加: $\mathrm{Cu} / \mathrm{Zn} / \mathrm{Al}<\mathrm{Cu} / \mathrm{Zn} / \mathrm{Al} / \mathrm{Zr}<\mathrm{Cu} / \mathrm{Zn} / \mathrm{Al} / \mathrm{Y}<\mathrm{Cu} /$ $\mathrm{Zn} / \mathrm{Al} / \mathrm{Zr} / \mathrm{Y}$. 甲醇选择性也随 $\mathrm{Zr}$ 和 $\mathrm{Y}$ 助剂的引入显 著增加, 并随强碱位比例的增加而线性增加, $\mathrm{Zr}$ 对 甲醇选择性的提高更明显. 因而, 少量 $\mathrm{Zr}$ 和 $\mathrm{Y}$ 助剂 的引入就可以促进甲醇的合成, 两种助剂共同修饰 的 $\mathrm{Cu} / \mathrm{Zn} / \mathrm{Al} / \mathrm{Zr} / \mathrm{Y}$ 催化剂上的甲醇收率最高, 达到 $0.52 \mathrm{~g} \cdot \mathrm{g}^{-1} \cdot \mathrm{h}^{-1}$.

\section{References}

(1) Choudhury, J. ChemCatChem 2012, 4 (5), 609. doi: 10.1002/ cctc. 201100495

(2) Olah, G. A.; Geoppert, A.; Prakash, G. K. S. Beyond Oil and Gas: the Methanol Economy, 1st ed.; Wiley-VCH: Weinheim, 2006; pp 173-187, 239-245.

(3) Pontzen, F.; Liebner, W.; Gronemann, V.; Rothaemel, M.; Ahlers, B. Catal. Today 2011, 171 (1), 242. doi: 10.1016/j. cattod.2011.04.049

(4) Yang, R. Q.; Yu, X. C.; Zhang, Y.; Li, W. Z.; Tsubaki, N. Fuel 2008, 87 (4-5), 443. doi: 10.1016/j.fuel.2007.06.020

(5) Jun, K. W.; Shen, W. J.; Rao, K. S. R.; Lee, K. W. Appl. Catal. A: Gen. 1998, 174, 231. doi: 10.1016/S0926-860X(98)00195-1

(6) Hong, Z. S.; Cao, Y.; Deng, J. F.; Fan, K. N. Catal. Lett. 2002, $82(1-2), 37$

(7) Gao, P.; Li, F.; Zhang, L. N.; Zhao, N.; Xiao, F. K.; Wei, W.; Zhong, L. S.; Sun, Y. H. Journal of $\mathrm{CO}_{2}$ Utilization 2013, 2 , 16. doi: 10.1016/j.jcou.2013.06.003

(8) Wang, J.; Chen, H. B.; Yun, H.; Lin, J. D.; Yi, J.; Zhang, H. B.; Liao, D. W. Acta Phys. -Chim. Sin. 2003, 19 (1), 65. [王 进, 陈鸿博, 云 虹, 林敬东, 易 军, 张鸿斌, 廖代伟. 物理化学学 报, 2003, 19 (1), 65.] doi: 10.3866/PKU.WHXB20030115

(9) Gao, P.; Li, F.; Zhan, H. J.; Zhao, N.; Xiao, F. K.; Wei, W.; Zhong, L. S.; Wang, H.; Sun, Y. H. J. Catal. 2013, 298, 51. doi: 10.1016/j.jcat.2012.10.030

(10) An, X.; Li, J. L.; Zuo, Y. Z.; Zhang, Q.; Wang, D. Z.; Wang, J. F. Catal. Lett. 2007, 118 (3-4), 264. doi: 10.1007/s10562-0079182-x

(11) Fernandez, J. M.; Barriga, C.; Ulibarri, M. A.; Labajos, F. M.; Rives, V. Chem. Mater. 1997, 9 (1), 312. doi: 10.1021/ cm9603720

(12) Zhang, L. H.; Li, F.; Evans, D. G.; Duan, X. Mater. Chem. Phys. 2004, 87 (2-3), 402. doi: 10.1016/j.matchemphys.2004.06.010

(13) Alejandre, A.; Medina, F.; Salagre, P.; Correig, X.; Sueiras, J. E. Chem. Mater 1999, 11 (4), 939. doi: 10.1021/cm980500f

(14) Zhang, L. H.; Zheng, C.; Li, F.; Evans, D. G.; Duan, X. J. Mater. Sci. 2008, 43 (1), 237. doi: 10.1007/s10853-007-2167-8

(15) Gao, P.; Li, F.; Zhao, N.; Xiao, F. K.; Wei, W.; Zhong, L. S.;
Sun, Y. H. Appl. Catal. A: Gen. 2013, 468, 442. doi: 10.1016/j. apcata.2013.09.026

(16) Shannon, R. D. Acta Crystallogr. A 1976, 32, 751. doi: 10.1107/ S0567739476001551

(17) Wu, G. D.; Wang, X. L.; Chen, B.; Li, J. P.; Zhao, N.; Wei, W.; Sun, Y. H. Appl. Catal. A: Gen. 2007, 329, 106. doi: 10.1016/j. apcata.2007.06.031

(18) Velu, S.; Sabde, D. P.; Shah, N.; Sivasanker, S. Chem. Mater. 1998, 10 (11), 3451. doi: 10.1021/cm980185x

(19) Zhang, L. H.; Li, F.; Evans, D. G.; Duan, X. Ind. Eng. Chem. Res. 2010, 49 (13), 5959. doi: 10.1021/ie9019193

(20) Gao, P.; Li, F.; Xiao, F. K.; Zhao, N.; Sun, N. N.; Wei, W.; Zhong, L. S.; Sun, Y. H. Catal. Sci. Technol. 2012, 2 (7), 1447. doi: 10.1039/c2cy00481j

(21) Cheng, J.; Yu, J. J.; Wang, X. P.; Li, L. D.; Li, J. J.; Hao, Z. P. Energy Fuels 2008, 22 (4), 2131. doi: 10.1021/ef8000168

(22) Xu, Z. P.; Zeng, H. C. J. Phys. Chem. B 2000, 104 (44), 10206. doi: 10.1021/jp001963n

(23) Trujillano, R.; Holgado, M. J.; Pigazo, F.; Rives, V. Physica B 2006, 373 (2), 267. doi: 10.1016/j.physb.2005.11.154

(24) Behrens, M.; Kasatkin, I.; Kuhl, S.; Weinberg, G. Chem. Mater. 2010, 22 (2), 386. doi: 10.1021/cm9029165

(25) Gao, P.; Li, F.; Xiao, F.; Zhao, N.; Wei, W.; Zhong, L. S.; Sun, Y. H. Catal. Today 2012, 194 (1), 9. doi: 10.1016/j. cattod.2012.06.012

(26) Guo, X. M.; Mao, D. S.; Lu, G. Z.; Wang, S.; Wu, G. S. J. Catal. 2010, 271, 178. doi: 10.1016/j.jcat.2010.01.009

(27) Guo, X. M.; Mao, D. S.; Lu, G. Z.; Wang, S. Acta Phys. -Chim. Sin. 2012, 28 (1), 170. [ 郭晓明, 毛东森, 卢冠 忠, 王 嵩. 物理化学学报, 2012, 28 (1), 170.] doi: 10.3866/ PKU.WHXB201228170

(28) Arena, F.; Italiano, G.; Barbera, K.; Bordiga, S.; Bonura, G.; Spadaro, L.; Frusteri, F. Appl. Catal. A: Gen. 2008, 350, 16. doi: 10.1016/j.apcata.2008.07.028

(29) Guo, X.; Mao, D.; Lu, G.; Wang, S.; Wu, G. J. Mol. Catal. A: Chem. 2011, 345 (1-2), 60. doi: 10.1016/j.molcata.2011.05.019

(30) Wilmer, H.; Genger, T.; Hinrichsen, O. J. Catal. 2003, 215, 188 doi: 10.1016/S0021-9517(03)00003-4

(31) Waugh, K. C. Solid State Ionics 2004, 168 (3-4), 327. doi: 10.1016/j.ssi.2003.05.001

(32) Wu, G. D.; Wang, X. L.; Wei, W.; Sun, Y. H. Appl. Catal. A: Gen. 2010, 377, 107. doi: 10.1016/j.apcata.2010.01.023

(33) Liu, Y. X.; Sun, K. P.; Ma, H. W.; Xu, X. L.; Wang, X. L. Catal. Commun. 2010, 11 (10), 880. doi: 10.1016/j.catcom.2010.03.014

(34) Liu, X. M.; Lu, G. Q.; Yan, Z. F.; Beltramini, J. Ind. Eng. Chem. Res. 2003, 42 (25), 6518. doi: 10.1021/ie020979s

(35) Arena, F.; Barbera, K.; Italiano, G.; Bonura, G.; Spadaro, L.; Frusteri, F. J. Catal. 2007, 249, 185. doi: 10.1016/j. jcat.2007.04.003

(36) Gao, L. Z.; Au, C. T. J. Catal. 2000, 189, 1. doi: 10.1006/ jcat.1999.2682 